\title{
PRESENCIA DE UROMYCES LIMONII (DC) LÉV. (ROYA DEL LIMONIUM): PRIMER REGISTRO PARA EL VALLE DE LLUTA, REGIÓN DE ARICA Y PARINACOTA
}

\author{
PRESENCE OF UROMYCES LIMONII (DC) LEV.: FIRST REGISTER FOR THE \\ LLUTA VALLEY-REGION OF ARICA AND PARINACOTA
}

Pía D. García-Hernández ${ }^{1}$; Mónica Rojas-Jara ${ }^{1}$; Germán F. Sepúlveda-Chavera ${ }^{1}$

\begin{abstract}
RESUMEN
Por primera vez es registrada la presencia de Uromyces limonii (DC) Lév, roya del Limonium, en el valle de Lluta, Región de Arica y Parinacota.

Palabras Clave: Uredinales, royas, micología.
\end{abstract}

ABSTRACT

Limonium rust [Uromyces limonii (DC) Lév.] is recorded for the first time from the Lluta valley region, northern Chile. Key words: Uredinales, rust, mycology.

\section{INTRODUCCIÓN}

La vegetación natural del valle de Lluta se compone de pocas especies que se repiten indefinidamente generando un aspecto "fitosociológico muy monótono", correspondiendo a formaciones de valles costeros, sometidos a condiciones climáticas y ecológicas propias de un clima desértico (Zollner, 1972).

Limonium spp es una planta rústica cultivada como flor de corte, que se encuentra principalmente en el sector medio del valle de Lluta. Durante el año 2006 se realizó una prospección fitogeográfica enmarcada en un estudio descriptivo y transeccional de Uredinales presentes en plantas silvestres y cultivadas de la región. En el hospedero estudiado se observaron síntomas característicos de roya en hojas y tallos de la planta y se estudiaron las estructuras taxonómicamente relevantes del hongo.

\section{MATERIALES Y MÉTODOS}

Se realizaron prospecciones en el sector medio del valle de Lluta. Para sistematizar la información generada, las diferentes localidades en donde se colectó fueron georreferenciadas con la ayuda de un aparato de Sistema de Posicionamiento Geográfico (GPS-eTrex Vista, Garmin Corp.), indicando aspectos como coordenadas geográficas y altitud.

El material vegetal colectado fue incorporado a la Colección Fitopatológica de la Universidad de Tarapacá (HFUTA: $\mathrm{N}^{\circ} 006$ ). Antes de ser incluido en la colección el material vegetal fue prensado y deshidratado por 3-4 días a $50{ }^{\circ} \mathrm{C}$. A partir de él se montaron exsicatas, rotuladas e identificadas con las informaciones geográficas y biológicas básicas.

La identificación de los taxa fúngicos se realizó teniendo como base los conceptos propuestos por

1 Laboratorio de Fitopatología, Departamento de Recursos Ambientales, Facultad de Ciencias Agronómicas, Universidad de Tarapacá. Avda. General Velásquez 1775, Arica, Chile. E-mail: gsepulve@uta.cl 
Cummins e Hiratzuka (1996), Saccardo (1888,1902), Lindquist (1951) y las descripciones de Oehrens (1972) y Mujica y Vergara (1980). Las técnicas utilizadas en la identificación y montaje del taxón fúngico incluido en este trabajo corresponden a técnicas rutinarias usadas en micología. Los signos fueron determinados mediante una lupa estereoscópica y fueron descritas las características de las colonias en las hojas, así como la disposición epífila, hipófila o anfígena de los soros. Las estructuras de valor taxonómico del hongo fueron estudiadas, documentadas y medidas utilizando un microscopio óptico compuesto (Fotomicroscopio II, Carl Zeiss Oberkochen, Alemania).

\section{RESULTADOS Y DISCUSIÓN}

La presencia de la enfermedad fue constante durante todo el año, manifestándose a través de pústulas rojizas correspondientes a los estadíos uredospóricos y teliospóricos contenidos en uredos y telios. Las características que presentan los ejemplares lluteños son: 1) uredos anfígenos de forma irregular, de color canela (Figura 1A); 2) uredosporas elipsoidales o globosas, finamente equinuladas
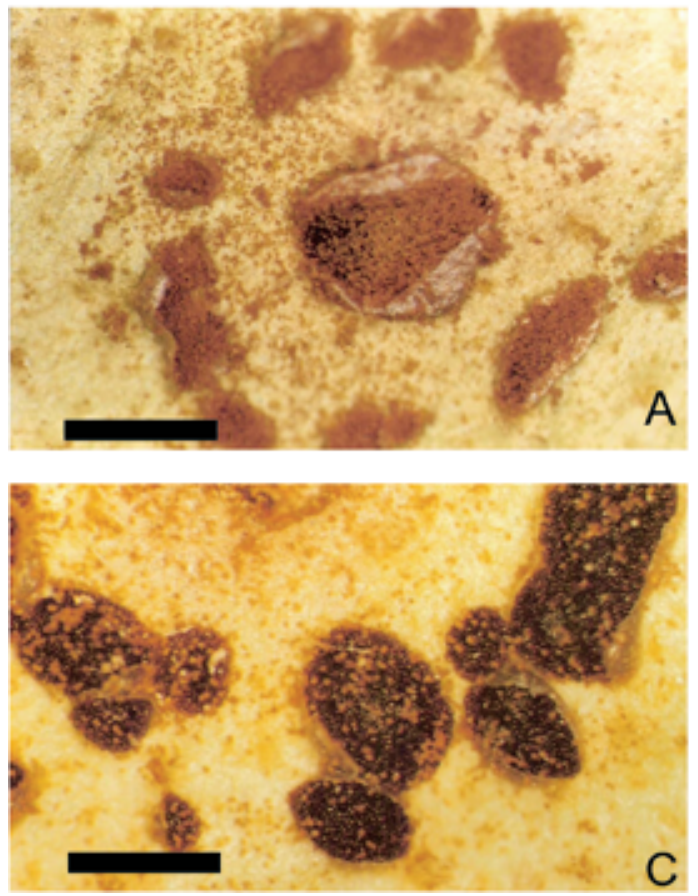

(Figura 1B), con episporio grueso, midiendo 24-27,6 x 24-28,8 $\mu \mathrm{m}$; los telios se presentan de forma anfígena, muy similares a los uredos, pero más oscuros y compactos (Figura 1C); 3) teliosporas de forma oblonga a elipsoides, midiendo 18-22,8 x 24-33,6 $\mu \mathrm{m}$, de pared externa lisa, color café-claro siendo más oscura distalmente, pedicelo hialino dos veces el largo de la espora (Figura 1D); episporio grueso, café-oscuro midiendo $6 \mu \mathrm{m}$ de espesor; aecios y picnidios ausentes. Basándose en las características morfológicas descritas, la roya corresponde a Uromyces limonii (DC.) Lév.

Uromyces limonii fue descrito por primera vez en 1849. De acuerdo con Lindquist (1951), corresponde a una especie cosmopolita. En Chile ha sido reportado en Tierra del Fuego para Armeria sp. (Mujica et al., 1980). Este reporte corresponde a la primera mención del hongo en el valle de Lluta, el más septentrional de la Región de Arica y Parinacota y del país.

\section{AGRADECIMIENTOS}

Los autores agradecen al Departamento de Recursos Ambientales de la Facultad de Ciencias
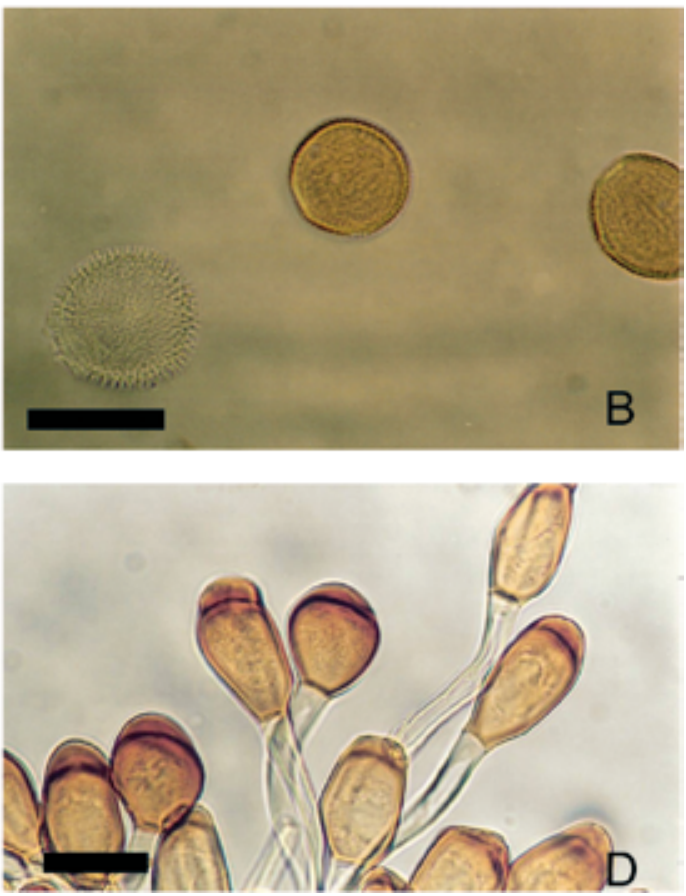

Figura 1. A: Uredos anfígenos en folíolo de Limonium spp.; B: Uredosporas; C: Telios anfígenos; D: Teliosporas de Uromyces limonii. Barras: A y $\mathrm{C}=3 \mathrm{~mm} ; \mathrm{B}=25 \mu \mathrm{m} ; \mathrm{D}=20 \mu \mathrm{m}$. 
Agronómicas y a la Dirección de Investigación y Postgrado de la Universidad de Tarapacá, Arica, Chile, por el soporte financiero prestado a través del

\section{LITERATURA CITADA}

CUMMINS, G.; HIRATSUKA, Y. 1996. Illustrated Genera of Rust Fungi. Edition. The American Phytopathological Society. St. Paul, Minnesota, USA. 152 pp.

LINDQUIST, J. C. 1951. Uredinales de la Provincia de Mendoza. Rev. Fac. Agr. Univ. La Plata. Vol. 28:65-114 pp.

MUJICA, R. F. \& VERGARA, C. C. 1980. Flora Fungosa Chilena. Revisada y Actualizada por Edgard Oehrens. $2^{\mathrm{a}}$ Edición. Universidad de Chile, Fac. de Agronomía, Cs. Agrícolas. Santiago de Chile. 124 pp.
Proyecto Mayor-UTA 7940 "Riqueza y Endemismo Micológico del Parque Nacional Lauca, I Región de Chile".

OEHRENS, E. 1972. Hongos Fitopatógenos del Departamento de Arica (I). Est. Exp. Agron. IDESIA 2: 6-32.

SACCARDO, P. A. 1888. Sylloge Fungorum omnium hucusque cognitorum. Uromyces limonii (DC.) Lév. Vol. VII: 532-533 pp.

ZOLLNER, O. 1972. Vegetación Natural del Valle de Azapa. IDESIA $\mathrm{N}^{\circ} 2$, Universidad del Norte, Arica-Chile. $117 \mathrm{pp}$. 
